\title{
Comparação entre a Medida Contínua do Débito Cardíaco e por Termodiluição em Bolus durante a Revascularização Miocárdica Sem Circulação Extracorpórea *
}

\section{Continuous and Bolus Thermodilution Cardiac Output Measurement during Off-Pump Coronary Artery Bypass Surgery}

Sílvia M. Kim ${ }^{1}$; Sílvia D. S. Oliveira ${ }^{2}$; Ubirajara S. Fonseca ${ }^{2}$; Luiz Marcelo Sá Malbouisson, TSA ${ }^{3}$; José Otávio Costa Auler Júnior, TSA ${ }^{4}$; Maria José Carvalho Carmona TSA ${ }^{5}$

\begin{abstract}
RESUMO
Kim SM, Oliveira SDS, Fonseca US, Malbouisson LMS, Auler Jr JOC, Carmona MJC - Comparação entre a Medida Contínua do Débito Cardíaco e por Termodiluição em Bolus durante a Revascularização Miocárdica Sem Circulação Extracorpórea
\end{abstract}

JUSTIFICATIVA E OBJETIVOS: A cirurgia de revascularização miocárdica sem o uso de circulação extracorpórea (CEC) relaciona-se a importantes alterações hemodinâmicas bruscas, que podem não ser prontamente detectadas pela medida contínua de débito cardíaco. Este estudo compara resultados obtidos pela medida do índice cardíaco com o cateter de artéria pulmonar com filamento térmico (Baxter Edwards Critical Care, Irvine, CA) com o método padrão por termodiluição com solução, durante a anastomose coronariana distal.

MÉTODO: Dez pacientes submetidos à cirurgia de revascularização miocárdica sem CEC foram monitorizados com o cateter de artéria pulmonar com filamento térmico. As medidas de índice cardíaco foram obtidas em quatro momentos: no início da anestesia, enquanto o tórax ainda estava fechado $\left(M_{1}\right)$, após a esternotomia $\left(M_{2}\right)$, após a estabilização do coração com o aparelho octopus $\left(M_{3}\right)$ e ao final da anastomose coronariana distal $\left(M_{4}\right)$.

RESULTADOS: Houve diminuição significativa $(p<0,05)$ do índice cardíaco durante a anastomose coronariana, detectada pela medida com termodiluição com bolus de solução. O índice cardíaco variou de $2,8 \pm 0,7$ para $2,3 \pm 0,8 \mathrm{l} . \mathrm{min} \cdot \mathrm{m}^{-2}$ no início da anastomose e 2,5 \pm 0,8 l. min. $\mathrm{m}^{-2}$ ao final da mesma. Essa

* Recebido do (Received from) Serviço de Anestesiologia e Divisão de Cirurgia do Instituto do Coração do Hospital das Clínicas da Faculdade de Medicina da Universidade de São Paulo (HC - FMUSP), SP

1. Preceptora da Disciplina de Anestesiologia da FMUSP

2. ME do CET/SBA do HC da FMUSP

3. Doutor em Anestesiologia pela FMUSP. Assistente do Serviço de Anestesiologia e Terapia Intensiva Cirúrgica do Instituto do Coração do HC da FMUSP

4. Professor Titular da Disciplina de Anestesiologia da FMUSP; Diretor do Serviço de Anestesiologia e Terapia Intensiva Cirúrgica do Instituto do Coração do HC da FMUSP

5. Professora Doutora da Disciplina de Anestesiologia da FMUSP; Supervisora do Serviço de Anestesiologia e Terapia Intensiva Cirúrgica do Instituto do Coração do HC da FMUSP

Apresentado (Submitted) em 12 de maio de 2003

Aceito (Accepted) para publicação em 20 de agosto de 2003

Endereço para correspondência (Correspondence to)

Dra. Maria José Carvalho Carmona

Rua Rodésia, 161/82 Vila Madalena

05435-020 São Paulo, SP

E-mail: maria.carmona@incor.usp.br

(c) Sociedade Brasileira de Anestesiologia, 2004 variação não foi detectada pela medida contínua (de $3 \pm 0,6$ para $3,2 \pm 0,5$ e $3,1 \pm 0,6$ l.min. $m^{-2}$ durante a anastomose coronariana).

CONCLUSÕES: A medida de débito cardíaco contínuo utilizando o cateter de artéria pulmonar com filamento térmico apresentou atraso na detecção das alterações hemodinâmicas agudas relacionadas à mudança do posicionamento do coração na cirurgia de revascularização miocárdica sem CEC.

Unitermos: CIRURGIA, Cardíaca: revascularização do miocárdio; MONITORIZAÇÃO: débito cardíaco; TÉCNICAS DE MEDIÇÃO: termodiluição

\section{SUMMARY}

Kim SM, Oliveira SDS, Fonseca US, Malbouisson LMS, Auler Jr JOC, Carmona MJC - Continuous and Bolus Thermodilution Cardiac Output Measurement during Off-Pump Coronary Artery Bypass Surgery

BACKGROUND AND OBJECTIVES: Off-pump CABG surgery is related to major and abrupt hemodynamic changes that may not be immediately detected by continuous cardiac output measurement (CCO). This study aimed at comparing results of cardiac index measurement with pulmonary artery catheter (PAC) with thermal filament (Baxter Edwards Critical Care, Irvine, CA) versus standard bolus thermodilution method during distal coronary anastomosis.

METHODS: Participated in this study 10 patients undergoing off-pump CABG who were monitored with PAC with thermal filament. Measurements of cardiac index were obtained in four moments: at anesthetic induction with the chest still closed $\left(M_{1}\right)$, after sternotomy $\left(M_{2}\right)$, after heart stabilization with the octopus device $\left(M_{3}\right)$ and at distal anastomosis completion $\left(M_{4}\right)$.

RESULTS: There has been significant cardiac index decrease $(p<0.05)$ during coronary anastomosis, detected when measurements were taken with bolus thermodilution method. Cardiac index has varied $2.8 \pm 0.7$ to $2.3 \pm 0.8$ L.min. m $^{-2}$ in the beginning and $2.5 \pm 0.8$ L.min. $\mathrm{m}^{-2}$ at the end of anastomosis. This variation was not detected by the continuous method (from $3 \pm 0.6$ to $3.2 \pm 0.5$ to $3.1 \pm 0.6$ L.min. $\mathrm{m}^{-2}$ during anastomosis). CONCLUSIONS: CCO measurement with PAC was late in detecting acute hemodynamic changes due to changes in heart position during off-pump CABG.

Key Words: MEASUREMENT TECHNIQUES: thermodilution; MONITORING: cardiac output; SURGERY, Cardiac: coronary artery bypass

\section{INTRODUÇÃO}

cirurgia de revascularização miocárdica sem circulação lextracorpórea (CEC) pode provocar alterações hemodinâmicas bruscas e transitórias relacionadas ao posiciona- 
mento do coração e a alterações isquêmicas. O posicionamento não-anatômico do coração, para facilitar a exposição das artérias a serem anastomosadas, pode levar à diminuição do débito cardíaco. A monitorização hemodinâmica tem, assim, papel importante no reconhecimento da diminuição do débito cardíaco ${ }^{1-7}$.

Monitores capazes de acompanhar continuamente o débito cardíaco foram desenvolvidos e introduzidos na prática clínica. O cateter de artéria pulmonar com termofilamento baseiase na emissão de energia térmica para calcular o débito cardíaco pelo princípio de termodiluição. Durante a anastomose da artéria torácica interna à artéria descendente anterior, as medidas contínuas mostraram boa correlação com as condições hemodinâmicas reais ${ }^{8}$, mas os resultados obtidos pelo método contínuo e por termodiluição em bolus apresentam diferenças que não podem ser aceitas clinicamente ${ }^{9}$.

Este estudo tem como objetivo identificar as alterações do débito cardíaco que ocorrem no posicionamento do coração, durante a cirurgia, e comparar os resultados obtidos pelo método de termodiluição contínuo e intermitente, com injeção de bolus de solução.

\section{MÉTODO}

O protocolo foi aprovado pela Comissão Científica do Instituto do Coração (InCor) e pelo Comitê de Análise de Projetos de Pesquisa (CAPPesq) do Hospital das Clínicas da Faculdade de Medicina da USP.

Foram avaliados dez pacientes de ambos os sexos, com idades entre 48 e 78 anos, submetidos à cirurgia de revascularização miocárdica sem CEC. O risco cirúrgico foi estabelecido segundo os critérios de Higgins ${ }^{10}$, sendo admitidos pacientes com risco mínimo a moderado. As artérias coronárias revascularizadas foram a descendente anterior, marginal esquerda, diagonal ou circunflexa. Para este estudo, avaliaram-se apenas as alterações hemodinâmicas ocorridas até a realização da primeira anastomose.

Todos os pacientes foram avaliados no dia anterior à cirurgia e receberam midazolam $7,5 \mathrm{mg}$ por via oral no momento do encaminhamento ao centro cirúrgico. Após monitorização com eletrocardiografia nas derivações $D_{\|}$e V5, com análise contínua de segmento ST e oximetria de pulso, foram submetidos à venóclise periférica com cateter calibroso e canulização de artéria radial. Iniciou-se a indução da anestesia geral com a administração titulada de sufentanil até uma dose de $0,5 \mu \mathrm{g} \cdot \mathrm{kg}^{-1}$ e propofol (até a perda dos reflexos) ou etomidato $\left(0,2 \mathrm{mg} \cdot \mathrm{kg}^{-1}\right)$. O relaxamento muscular foi obtido com pancurônio $\left(0,08 \mathrm{mg} \cdot \mathrm{kg}^{-1}\right)$ ou atracúrio $(0,5$ $\left.\mathrm{mg} \cdot \mathrm{kg}^{-1}\right)$ e a intubação orotraqueal foi realizada após ventilação manual sob máscara. A anestesia foi mantida com concentrações variáveis de isoflurano $(0,7 \%$ a $0,9 \%)$ e boIus intermitentes de sufentanil.

O cateter de artéria pulmonar calibre 7F com filamento térmico (CCO catheter, Baxter Edwards Critical Care, Irvine, CA) foi introduzido na veia jugular interna direita e conectado ao monitor Vigilance (Baxter Edwards Critical Care, Irvine, CA).
Após o posicionamento do cateter na artéria pulmonar, foi coIhida amostra de sangue venoso misto para realização de exame de gasometria e calibração do monitor de saturação venosa contínua.

A avaliação hemodinâmica foi realizada após a indução anestésica e com o tórax ainda fechado $\left(\mathrm{M}_{0}\right)$, após a esternotomia $\left(\mathrm{M}_{1}\right)$, no início da anastomose coronariana, com o dispositivo estabilizador da parede miocárdica Octopus (Medtronic, Inc., Minneapolis, Minn.) posicionado $\left(\mathrm{M}_{2}\right)$ e ao final da anastomose, antes da retirada do Octopus $\left(\mathrm{M}_{3}\right)$. As medidas de débito cardíaco foram divididas pela medida de superfície corpórea para obtenção do valor de índice cardíaco.

O valor de débito cardíaco registrado pelo monitor Vigilance nos momentos estudados foi considerado como débito contínuo. Em seguida, as medidas de débito cardíaco foram realizadas com injeção de bolus de $10 \mathrm{ml}$ de solução fisiológica em temperatura ambiente. As curvas de termodiluição foram avaliadas e o resultado registrado considerou a média de três medidas consecutivas.

Os dados foram avaliados utilizando-se o método de Análise de Variância de duas vias para medidas repetidas (ANOVA). $O$ valor de $p<0,05$ foi considerado estatisticamente significativo.

\section{RESULTADOS}

Os dados antropométricos relacionando sexo, idade, superfície corpórea, risco cirúrgico e local da anastomose coronariana são apresentados na tabela I. Na tabela Il estão os valores de índice cardíaco obtidos pela técnica contínua e de termodiluição intermitente e da saturação venosa mista de oxigênio $\left(\mathrm{SvO}_{2}\right)$, também representados na figura 1 .

Tabela I - Dados Antropométricos, Risco Cirúrgico e Primeira Anastomose

\begin{tabular}{lccccl}
\hline & Sexo & $\begin{array}{c}\text { Idade } \\
(\text { anos })\end{array}$ & $\begin{array}{c}\text { SC } \\
\left(\mathrm{m}^{2}\right)\end{array}$ & $\begin{array}{c}\text { Risco } \\
\text { Cirúrgico }\end{array}$ & $\begin{array}{l}\text { Primeira Anastomose } \\
\text { Artérias Coronárias }\end{array}$ \\
\hline Caso 1 & $\mathrm{F}$ & 48 & 1,92 & 1 & Descendente Anterior \\
Caso 2 & $\mathrm{M}$ & 52 & 1,67 & 2 & Marginal Esquerda \\
Caso 3 & $\mathrm{M}$ & 60 & 2,04 & 5 & Diagonal \\
Caso 4 & $\mathrm{M}$ & 65 & 1,76 & 5 & Diagonal \\
Caso 5 & $\mathrm{M}$ & 67 & 1,46 & 3 & Marginal Esquerda \\
Caso 6 & $\mathrm{F}$ & 70 & 1,73 & 2 & Circunflexa \\
Caso 7 & $\mathrm{F}$ & 72 & 1,61 & 5 & Circunflexa \\
Caso 8 & $\mathrm{M}$ & 64 & 1,75 & 1 & Descendente Anterior \\
Caso 9 & $\mathrm{F}$ & 75 & 1,46 & 4 & Diagonal \\
Caso 10 & $\mathrm{M}$ & 78 & 1,69 & 3 & Marginal Esquerda \\
\hline
\end{tabular}

SC: superfície corpórea 
Tabela II - Índice Cardíaco e $\mathrm{SvO}_{2}$ (Média \pm DP)

\begin{tabular}{llccc}
\hline & & Bolus & Contínuo & $\mathrm{SvO}_{2}$ \\
\hline $\mathrm{M}_{0}$ & Tórax fechado & $2,2 \pm 0,7$ & $2,6 \pm 0,7$ & $77,1 \pm 5,7$ \\
$\mathrm{M}_{1}$ & Tórax aberto & $2,8 \pm 0,7$ & $3,0 \pm 0,6$ & $81,6 \pm 5,3$ \\
$\mathrm{M}_{2}$ & Início da anastomose & $2,3 \pm 0,8$ & $3,2 \pm 0,5^{*}$ & $72,3 \pm 12,3$ \\
$\mathrm{M}_{3}$ & Final da anastomose & $2,5 \pm 0,8$ & $3,1 \pm 0,6^{*}$ & $71,2 \pm 12,8$ \\
\hline
\end{tabular}

${ }^{*} p<0,05$

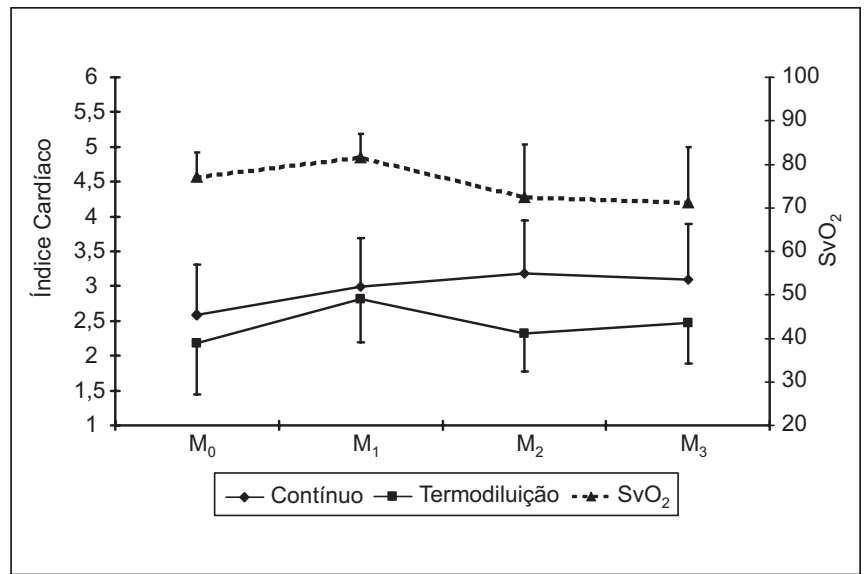

Figura 1 - Comparação entre as Medidas de Índice Cardíaco pelo Método Contínuo e de Termodiluição com Bolus e $\mathrm{SvO}_{2}$ $\left(\mathrm{M}_{0}\right.$ - tórax fechado, $\mathrm{M}_{1}$ - tórax aberto, $\mathrm{M}_{2}$ - início da anastomose, $\mathrm{M}_{3}$ - final da anastomose)

Todos os pacientes apresentaram diminuição do índice cardíaco durante a anastomose coronariana, detectada tanto por termodiluição contínua como intermitente. A diferença entre os valores médios detectados pelos dois métodos nos diferentes tempos cirúrgicos foi estatisticamente significativa $(p \leq 0,001)$. Não houve diferença estatisticamente significativa entre os valores obtidos pelos dois métodos nos momentos $\mathrm{M}_{0}$ (após a indução da anestesia) e $\mathrm{M}_{1}$ (tórax aberto). No entanto, houve diferença entre os valores $(p<0,05)$ nos momentos de diminuição abrupta do débito cardíaco, durante a realização das anastomoses coronarianas, tanto no início como no final do procedimento. A diminuição da $\mathrm{SvO}_{2}$ apresentou interação estatisticamente significativa com a diminuição do débito cardíaco detectada pela medida com termodiluição em bolus.

\section{DISCUSSÃO}

É cada vez mais freqüente a realização de cirurgias de revascularização miocárdica sem o uso da CEC, na tentativa de diminuir os seus efeitos adversos e de ter uma opção de cirurgia menos invasiva e com menor morbidade ${ }^{7}$. O emprego da CEC pode causar diversas alterações, como a síndrome da resposta inflamatória sistêmica, alterações da coagulação, hemodiluição e risco de embolia aérea, além de alterações da função pulmonar no período pós-operatório. Fatores como reoperação coronariana, idade avançada, sexo feminino e disfunção ventricular esquerda são fatores de previsão independentes do alto risco de mortalidade em cirurgias de revascularização miocárdica. A cirurgia sem CEC é uma possível alternativa para pacientes de alto risco ${ }^{11,12}$.

Para estabilizar e imobilizar a região da parede miocárdica a ser manipulada, são utilizados atualmente dispositivos que facilitam a técnica cirúrgica e diminuem a necessidade do uso de fármacos para diminuir a freqüência cardíaca ${ }^{13}$. A incidência de complicações como conversão para CEC, infarto miocárdico e re-intervenção coronariana foram baixas, sugerindo que o uso do estabilizador de tecido Octopus é seguro ${ }^{14}$. Entretanto, podem ocorrer importantes alterações hemodinâmicas diretamente relacionadas às mudanças na posição do coração e manipulação ventricular para a realização da anastomose coronariana.

As flutuações hemodinâmicas podem ser diminuídas pelo ajuste da pré-carga e utilização de drogas vasoativas. É importante que as alterações sejam rapidamente detectadas para orientar a terapêutica a ser adotada. Mudanças na posição da mesa cirúrgica, administração de volume ou mesmo suporte farmacológico podem ser necessários para tratar a súbita diminuição no débito cardíaco.

Amedida contínua de débito cardíaco através do cateter com filamento térmico Edwards CCO catheter (Baxter Edwards Critical Care, Irvine, CA) representa interessante meio de monitorização, usando o princípio de termodiluição do sangue aquecido por pulsos de calor emitidos pelo filamento posicionado no ventrículo direito ${ }^{8}$.

Estudos clínicos e experimentais demonstraram boa correlação dos valores de débito cardíaco obtidos pela técnica de medida contínua com a medida intermitente com bolus de solução fria ${ }^{8}$. Outros estudos, no entanto, mostraram que há um atraso na detecção de alterações hemodinâmicas agudas ${ }^{15}$

Conforme observado neste estudo, houve boa correlação entre os valores de índice cardíaco obtidos pelo método padrão de termodiluição com bolus e pela medida contínua com cateter de artéria pulmonar com filamento térmico nos momentos de manutenção da situação hemodinâmica. No entanto, nos momentos em que houve alteração abrupta da função cardíaca, como durante a colocação do dispositivo estabilizador da parede ventricular e ao final da anastomose, houve atraso no registro de medida contínua. Com uma diminuição breve do débito cardíaco, não ocorreu alteração no valor registrado pelo monitor, que representou uma média dos valores obtidos nos três a seis minutos anteriores. Por esse motivo, alguns centros sugerem o uso do termo débito cardíaco semi-contínuo para descrever a técnica. A medida registrada no modo STAT é mais precisa, porém, mais lenta

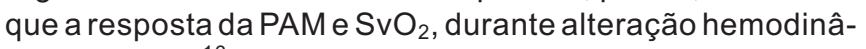
mica aguda ${ }^{16}$. No entanto, a intensa manipulação do coração no momento da medida poderia interferir ainda mais na técnica.

Essa desvantagem da monitorização contínua do débito cardíaco pode ser reduzida realizando-se medidas de débito cardíaco por termodiluição em bolus sempre que houver al- 
teração hemodinâmica aguda por sangramento intenso ou importante diminuição da $\mathrm{SvO}_{2}$. Do mesmo modo que durante hemorragia aguda a $\mathrm{SvO}_{2}$ decai paralelamente à diminuição do débito cardíaco ${ }^{15}$, a monitorização contínua da $\mathrm{SvO}_{2}$ acompanha diretamente as mudanças no débito cardíaco, considerando-se que a saturação arterial de oxigênio e os níveis de hemoglobina permaneçam constantes. Dessa forma, as alterações hemodinâmicas súbitas da revascularização miocárdica sem CEC podem justificar a perda de correlação nas duas técnicas de medidas de débito cardíaco.

Concluindo, a medida de débito cardíaco contínuo utilizando o cateter de artéria pulmonar com filamento térmico apresentou atraso na detecção das alterações hemodinâmicas agudas relacionadas à mudança do posicionamento do coração na cirurgia de revascularização miocárdica sem CEC. Portanto, neste tipo de cirurgia, nos momentos iniciais e finais da anastomose coronariana, é aconselhável a complementação da medida contínua de débito cardíaco com injeções em bolus ou com a monitorização da saturação venosa mista de oxigênio.

\section{Continuous and Bolus Thermodilution Cardiac Output Measurement during Off-Pump Coronary Artery Bypass Surgery}

Sílvia M. Kim, M.D.; Sílvia D. S. Oliveira, M.D.; Ubirajara S. Fonseca, M.D.; Luiz Marcelo Sá Malbouisson, TSA, M.D.; José Otávio Costa Auler Júnior, TSA, M.D.; Maria José Carvalho Carmona TSA, M.D.

\section{INTRODUCTION}

Off-pump coronary artery bypass surgery (CABG) may promote sudden and transient hemodynamic changes related to heart positioning and ischemic changes. Non-anatomic heart position to help arteries exposure may decrease cardiac output. So, hemodynamic monitoring plays an important role in identifying decreased cardiac output ${ }^{1-7}$.

Monitors to continuously monitor cardiac output have been developed and introduced in the clinical practice. Pulmonary artery catheter with thermal filament is based on thermal energy emission to calculate cardiac output by the thermodilution principle. During internal thoracic artery anastomosis to anterior descending artery, continuous measurements have shown good correlation with actual hemodynamic conditions ${ }^{8}$, but results obtained with the continuous method and with bolus thermodilution have differences which cannot be clinically accepted ${ }^{9}$.

This study aimed at identifying cardiac output changes during intraoperative heart positioning and at comparing continuous and intermittent thermodilution method with solution bolus injection.

\section{METHODS}

The protocol was approved by the Scientific Committee, Instituto do Coração (InCor) and by the Research Projects Analysis Committee (CAPPesq), Hospital das Clínicas, Faculdade de Medicina, USP.

Participated in this study 10 patients of both genders, aged 48 to 78 years, submitted to off-pump coronary artery bypass surgery. Surgical risk was established according to Higgin's criteria ${ }^{10}$, being admitted low to moderate risk patients. Revascularized arteries were: anterior descending, left marginal, diagonal or circumflex. Only hemodynamic changes observed until first anastomosis were evaluated in this study. All patients were evaluated the day before surgery and received $7.5 \mathrm{mg}$ oral midazolam at referral to the operating center. After monitoring with $E C G$ at $D_{\|}$and $V 5$ leads with continuous ST segment analysis and pulse oximetry, patients were submitted to peripheral venoclysis with large catheter and radial artery catheterization. General anesthesia was induced with titrated sufentanil up to $0.5 \mu \mathrm{g} \mathrm{kg}^{-1}$ and propofol (until loss of reflexes) or etomidate $\left(0.2 \mathrm{mg} \cdot \mathrm{kg}^{-1}\right)$. Muscle relaxation was achieved with pancuronium $\left(0.08 \mathrm{mg} \cdot \mathrm{kg}^{-1}\right)$ or atracurium $\left(0.5 \mathrm{mg} . \mathrm{kg}^{-1}\right)$ and tracheal intubation was performed after manual ventilation under mask. Anesthesia was maintained with variable isoflurane concentrations $(0.7 \%$ to $0.9 \%$ ) and intermittent sufentanil bolus.

Pulmonary artery catheter (7F) with thermal filament (CCO catheter, Baxter Edwards Critical Care, Irvine, CA) was introduced in the right internal jugular vein and connected to a Vigilance monitor (Baxter Edwards Critical Care, Irvine, CA). After catheter positioning in the pulmonary artery, mixed venous blood sample was collected for gases analysis and continuous venous saturation monitor gaging.

Hemodynamic evaluation was performed after anesthetic induction and with the chest still closed $\left(\mathrm{M}_{0}\right)$, after sternotomy $\left(M_{1}\right)$, in the beginning of coronary anastomosis after myocardial wall stabilizing device Octopus (Medtronic, Inc., Minneapolis, Minn.) positioning $\left(\mathrm{M}_{2}\right)$ and at anastomosis completion before Octopus removal $\left(\mathrm{M}_{3}\right)$. Cardiac output measurements were divided by body surface measurements to obtain cardiac index value.

Cardiac output values recorded by the Vigilance monitor were considered continuous output. Then, cardiac output was measured with bolus injection of $10 \mathrm{~mL}$ saline solution in room temperature. Thermodilution curves were evaluated and recorded result has considered the mean of three consecutive measurements.

Data were evaluated by two-way Analysis of Variance for repetitive measurements (ANOVA), considering significant $p<$ 0.05 .

\section{RESULTS}

Anthropometric data relating gender, age, body surface, surgical risk and coronary anastomosis site are shown in table I. Table II shows cardiac index obtained by continuous and in- 
termittent thermodilution technique as well as mixed oxygen venous saturation $\left(\mathrm{SvO}_{2}\right)$, also represented in figure 1 .

Table I - Demographics Data, Surgical Risk and First Anastomosis

\begin{tabular}{lccccl}
\hline & Gender & $\begin{array}{c}\text { Age } \\
(\text { years })\end{array}$ & $\begin{array}{c}\text { BS } \\
\left(\mathrm{m}^{2}\right)\end{array}$ & $\begin{array}{c}\text { Surgical } \\
\text { Risk }\end{array}$ & $\begin{array}{l}\text { First Anastomosis } \\
\text { Coronary Arteries }\end{array}$ \\
\hline Case 1 & $\mathrm{F}$ & 48 & 1.92 & 1 & Anterior Descending \\
Case 2 & $\mathrm{M}$ & 52 & 1.67 & 2 & Left Marginal \\
Case 3 & $\mathrm{M}$ & 60 & 2.04 & 5 & Diagonal \\
Case 4 & $\mathrm{M}$ & 65 & 1.76 & 5 & Diagonal \\
Case 5 & $\mathrm{M}$ & 67 & 1.46 & 3 & Left Marginal \\
Case 6 & $\mathrm{F}$ & 70 & 1.73 & 2 & Circumflex \\
Case 7 & $\mathrm{F}$ & 72 & 1.61 & 5 & Circumflex \\
Case 8 & $\mathrm{M}$ & 64 & 1.75 & 1 & Anterior Descending \\
Case 9 & $\mathrm{F}$ & 75 & 1.46 & 4 & Diagonal \\
Case 10 & $\mathrm{M}$ & 78 & 1.69 & 3 & Left Marginal \\
\hline
\end{tabular}

BS: body surface

Table II - Cardiac Index and $\mathrm{SvO}_{2}$ (Mean $\pm \mathrm{SD}$ )

\begin{tabular}{llccc}
\hline & & Bolus & Continuous & $\mathrm{SvO}_{2}$ \\
\hline $\mathrm{M}_{0}$ & Closed chest & $2.2 \pm 0.7$ & $2.6 \pm 0.7$ & $77.1 \pm 5.7$ \\
$\mathrm{M}_{1}$ & Open chest & $2.8 \pm 0.7$ & $3.0 \pm 0.6$ & $81.6 \pm 5.3$ \\
$\mathrm{M}_{2}$ & Beginning of anastomosis & $2.3 \pm 0.8$ & $3.2 \pm 0.5^{*}$ & $72.3 \pm 12.3$ \\
$\mathrm{M}_{3}$ & End of anastomosis & $2.5 \pm 0.8$ & $3.1 \pm 0.6^{*}$ & $71.2 \pm 12.8$ \\
\hline
\end{tabular}

${ }^{*} p<0.05$

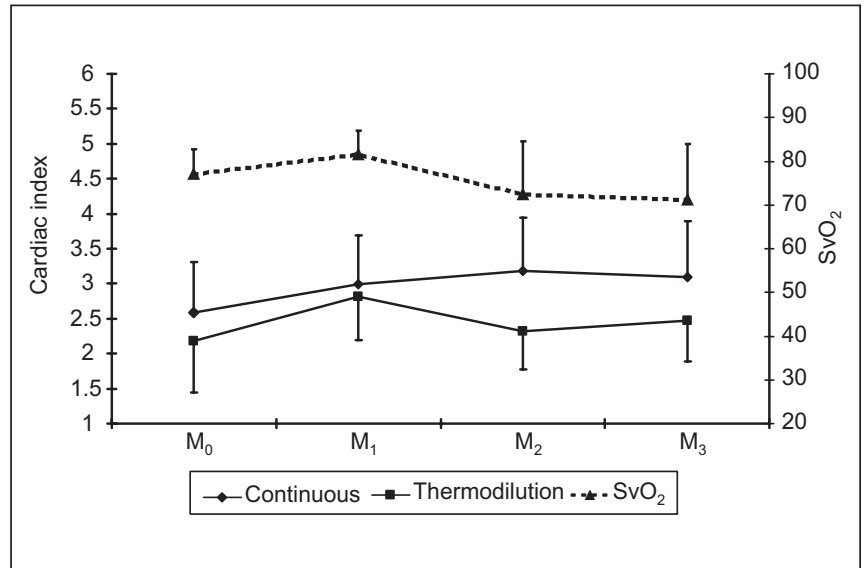

Figure 1 - Comparison of Cardiac Index Measurements by Continuous and Bolus Thermodilution Method and $\mathrm{SvO}_{2}$

$\left(\mathrm{M}_{0}\right.$ - closed chest, $\mathrm{M}_{1}$ - open chest, $\mathrm{M}_{2}$ - beginning of anastomosis, $\mathrm{M}_{3}$ - end of anastomosis)

All patients had decreased cardiac index during coronary anastomosis, detected both by continuous and intermitted thermodilution. Difference between mean values detected by both methods in different surgical times was statistically significant $(p \leq 0.001)$. There have been no statistically significant differences between values of both methods in moments $\mathrm{M}_{0}$ (after anesthetic induction) and $\mathrm{M}_{1}$ (open chest). How- ever, there have been differences between values $(p<0.05)$ at sudden cardiac output decrease during coronary anastomosis, both in beginning and end of procedure. $\mathrm{SvO}_{2}$ decrease has shown statistically significant interaction with cardiac output decrease detected by bolus thermodilution.

\section{DISCUSSION}

Off-pump coronary artery bypass surgery is becoming increasingly frequent, in an attempt to decrease the pump's adverse effects and having a less invasive option with lower morbidity ${ }^{7}$. Coronary artery bypass (CAB) may promote several changes, such as systemic inflammatory response syndrome, coagulation disorders, hemodilution and risk for air embolism, in addition to postoperative pulmonary function changes. Factors such as coronary reoperation, older age, female gender and left ventricular dysfunction are independent forecasters for high mortality risk during coronary artery bypass surgery. Off-pump surgery is a potential alternative for these high risk patients ${ }^{11,12}$. Currently, there are devices to stabilize and immobilize myocardial wall to be manipulated, which help surgical technique and decrease the need for heart rate-decreasing drugs ${ }^{13}$. The incidence of complications, such as $\mathrm{CAB}$ conversion, myocardial infarction and coronary re-intervention is low, suggesting that Octopus tissue stabilizer is a safe device ${ }^{14}$. However, there may be major hemodynamic changes directly related to changes in heart position and ventricular manipulation for coronary anastomosis.

Hemodynamic fluctuations may be minimized by preload adjustment and vasoactive drugs. It is important to early detect changes to orient therapy. Table position changes, volume administration or even drug support may be needed to manage sudden cardiac output decrease.

Continuous cardiac output measurement through Edwards CCO catheter thermal filament catheter (Baxter Edwards Critical Care, Irvine, CA) is an interesting monitoring means which uses the thermodilution principle of blood warmed by heat pulses emitted by the filament positioned in the right ventricle ${ }^{8}$.

Clinical and experimental studies have shown a good correlation between cardiac output values obtained by continuous measurement and intermittent measurements with cold solution bolus ${ }^{8}$. Other studies, however, have shown a delay in acute hemodynamic changes detection ${ }^{15}$.

As observed in our study, there has been good correlation between cardiac index values obtained by the standard bolus thermodilution method and by continuous measurements with pulmonary artery catheter with thermal filament in moments of hemodynamic status maintenance. However, in moments where there has been sudden cardiac function changes, such as during ventricular wall stabilizer device placement and at anastomosis completion, there has been continuous measurement delay. With brief cardiac output decrease there is no changes in monitor values, which represent mean values obtained in three to six previous minutes. For this reason, some centers suggest the term semi- 
continuous cardiac output to define the technique. Measurement recorded in the STAT mode is more accurate, however slower than MBP and $\mathrm{SvO}_{2}$ response during acute hemodynamic changes ${ }^{16}$. Nevertheless, massive heart manipulation during measurement could further interfere with the technique.

This continuous cardiac output monitoring disadvantage could be minimized by bolus thermodilution cardiac output measurements whenever there are acute hemodynamic changes by severe bleeding or major $\mathrm{SvO}_{2}$ decrease. As $\mathrm{SvO}_{2}$ during acute hemorrhage decreases in parallel with cardiac output decrease ${ }^{15}$, continuous $\mathrm{SvO}_{2}$ monitoring directly follows cardiac output changes, provided that oxygen arterial saturation and hemoglobin levels remain constant. This way, sudden hemodynamic changes of off-pump coronary artery bypass could justify the loss of correlation of both cardiac output measurement techniques.

In conclusion, continuous cardiac output measurement with pulmonary artery catheter with thermal filament was late in detecting acute hemodynamic changes related to changes in heart position during off-pump coronary artery bypass surgery. So, for this type of surgery, in initial and final anastomosis moments, it is recommended to complement continuous cardiac output measurement with bolus injections or with oxygen mixed venous saturation monitoring.

\section{REFERÊNCIAS - REFERENCES}

01. Torracca L, Schreuder JJ, Quarti A et al - Acute effects of beating heart coronary surgery on left ventricular performance. Ann Thorac Surg, 2002;74:S1348-1352.

02. Couture $P$, Denault A, Limoges $P$ et al - Mechanisms of hemodynamic changes during off-pump coronary artery bypass surgery. Can J Anaesth, 2002;49:835-849.

03. Do QB, Goyer C, Chavanon O et al - Hemodynamic changes during off-pump CABG surgery. Eur J Cardiothorac Surg, 2002;21:385-390.

04. D'Ancona G, Karamanoukian H, Lima R et al - Hemodynamic effects of elevation and stabilization of the heart during off-pump coronary surgery. J Card Surg, 2000;15:385-391.

05. Watters MP, Ascione R, Ryder IG et al - Haemodynamic changes during beating heart coronary surgery with the 'Bristol Technique'. Eur J Cardiothorac Surg, 2001;19:34-40.

06. Nierich AP, Diephuis J, Jansen EW et al - Heart displacement during off-pump CABG: how well is it tolerated? Ann Thorac Surg, 2000;70:466-472.

07. Heames RM, Gill RS, Ohri SK et al - Off-pump coronary artery surgery. Anaesthesia, 2002;57:676-685.

08. Singh A, Juneja R, Mehta Y et al - Comparison of continuous, stat, and intermittent cardiac output measurements in patients undergoing minimally invasive direct coronary artery bypass surgery. J Cardiothorac Vasc Anesth, 2002;16:186-190.

09. Zollner C, Goetz AE, Weis M et al - Continuous cardiac output measurements do not agree with conventional bolus thermodilution cardiac output determination. Can J Anaesth, 2001;48:1143-1147.

10. Higgins TL, Estafanous FG, Loop FD et al - Stratification of morbidity and mortality outcome by preoperative risk factors in coronary artery bypass patients. A clinical severity score. JAMA, 1992;267:2344-2348.
11. Stamou SC, Corso PJ - Coronary revascularization without cardiopulmonary bypass in high-risk patients: a route to the future. Ann Thorac Surg, 2001;71:1056-1061.

12. Cleveland Jr JC, Shroyer AL, Chen AY et al - Off-pump coronary artery bypass grafting decreases risk-adjusted mortality and morbidity. Ann Thorac Surg, 2001;72:1282-1288.

13. Bernardes CES, Messias ERR, Carmona MJC - Considerações anestésico-cirúrgicas sobre a revascularização do miocárdio através de minitoracotomia. Rev Bras Anestesiol, 1999;49:196-200.

14. Jansen EW, Borst C, Lahpor JR et al - Coronary artery bypass grafting without cardiopulmonary bypass using the octopus method: results in the first one hundred patients. J Thorac Cardiovasc Surg, 1998;116:60-67.

15. Poli de Figueiredo LF, Malbouisson LM, Varicoda EY et al - Thermal filament continuous thermodilution cardiac output delayed response limits its value during acute hemodynamic instability. J Trauma, 1999;47:288-293.

16. Lazor MA, Pierce ET, Stanley GD et al - Evaluation of the accuracy and response time of STAT-mode continuous cardiac output. J Cardiothorac Vasc Anesth, 1997;11:432-436.

\section{RESUMEN}

Kim SM, Oliveira SDS, Fonseca US, Malbouisson LMS, Auler Jr JOC, Carmona MJC - Comparación entre la Medida Continua del Débito Cardíaco y por Termodilución en Bolus durante la Revascularización Miocárdica sin Circulación Extracorpórea

JUSTIFICATIVA Y OBJETIVOS: La cirugía de revascularización miocárdica sin uso de circulación extracorpórea (CEC) se relaciona a importantes alteraciones hemodinámicas bruscas que pueden no ser prontamente detectadas por la medida continua de débito cardíaco. Este estudio compara resultados obtenidos por la medida del índice cardíaco con el catéter de arteria pulmonar con filamento térmico (Baxter Edwards Critical Care, Irvine, CA) con el método patrón por termodilución con solución, durante la anastomosis coronariana distal.

MÉTODO: Diez pacientes sometidos a la cirugía de revascularización miocárdica sin CEC fueron monitorizados con el catéter de arteria pulmonar con filamento térmico. Las medidas de índice cardíaco fueron obtenidas en cuatro momentos: al inicio de la anestesia, en cuanto el tórax aun estaba cerrado $\left(M_{1}\right)$, después de la esternotomia $\left(M_{2}\right)$, después de la estabilización del corazón con el aparato octopus $\left(M_{3}\right)$ y al final de la anastomosis coronariana distal $\left(M_{4}\right)$.

RESULTADOS: Hubo disminución significativa $(p<0,05)$ del índice cardíaco durante la anastomosis coronariana, detectada por la medida con termodilución con bolus de solución. El índice cardíaco varió de 2,8 $\pm 0,7$ para 2,3 $\pm 0,8$ L.min. $m^{-2}$ en el inicio de la anastomosis y $2,5 \pm 0,8$ L.min. $m^{-2}$ al final de la misma. Esa variación no fue detectada por la medida continua (de $3 \pm 0,6$ para $3,2 \pm 0,5$ y 3,1 $\pm 0,6$ L. min. m $^{-2}$ durante la anastomosis coronariana).

CONCLUSIONES: La medida de débito cardíaco continuo utilizando el catéter de arteria pulmonar con filamento térmico, presentó atraso en la detección de las alteraciones hemodinámicas agudas relacionadas al cambio del posicionamiento del corazón en la cirugía de revascularización miocárdica sin CEC. 